\title{
Bi-manual Interactive Tools for Cultural Heritage Researchers
}

\author{
Can Ozmen \\ Worcester Polytechnic Institute \\ Worcester, USA \\ canozmen@gmail.com
}

\author{
Selim Balcisoy \\ Sabanci University \\ Istanbul, Turkey \\ balcisoy@sabanciuniv.edu
}

\begin{abstract}
The availability of intuitive, user-friendly and specialized software to work with $3 D$ models of cultural heritage artifacts is as important as the availability of low-cost and robust data acquisition techniques for the adoption of digitized $3 D$ models in cultural heritage research. As recent developments in $3 D$ scanning technologies have made the digitization of artifacts affordable; the amount of digitized models available for research increases rapidly. Consequently the need for specialized software for cultural heritage research and practice on $3 D$ models becomes more apparent. The lack of spatial measurement tools familiar to cultural heritage experts in traditional $3 D$ modeling packages motivated us to create a simple, freely available, and extensible measurement tools system, $\mathrm{CH}$ Toolbox, which was designed exclusively for cultural heritage research. The proposed system visualizes digitized models of artifacts in $3 D$ and allows the user to analyze the pieces using a spaceball and mouse driven bi-manual interface. We describe here the components of the CH Toolbox system, specificially the virtual tape measure, caliper, rim chart and a surface area estimation tool. Additionally, we present justification for CH Toolbox's bi-manual interaction scheme according to Guiard's Kinematic Chain model for asymmetric human skilled bi-manual actions.
\end{abstract}

\section{Introduction}

This paper deals with the design and development of a software system to work with digitized 3D models of cultural heritage artefacts. The work done can be summarized as investigating the workflow of cultural heritage researchers, analyzing their needs and requirements from such a software package, analyzing the usage of the tools choosen for implementation, devising an interaction scheme to maximize the ease of use and adoption of the software system, and finally the implementation of the tools according to the requirements collected by adopting appropriate techniques used in other computational geometry tasks. Our contributions are :

- Introduction of bi-manual asymetric interaction to the computer assisted cultural heritage domain to work with high detailed digitized 3D models of artefacts.

- Development of computer-aided versions of four spatial measurement tools that archaeologists, art historians, and other cultural heritage workers use in their workflow by adopting algorithms for other computational geometry tasks. The tools include an interactive tape measure, caliper, rim chart, and surface area estimation.

This paper presents new tools and introduction of bi-manual interaction on top of our existing software system. The tape measure and rim chart have been introduced in [15], followed by [16] where the caliper was added. The paper starts with giving background information about the use of computer graphics in cultural heritage applications. The next chapter defines the research problem and presents our motivation. The asymetric bi-manual interaction scheme design is presented next, followed by detailed descriptions of the tools themselves. Finally the paper is finished with tests results, conluding remarks, and suggested topics for the future direction for this research.

\section{Background Information}

\subsection{Computer Graphics and Cultural Heritage}

The increase in computing power and its availability, combined with the interest in preserving and displaying items deemed part of humanity's cultural heritage has made cultural heritage an important application domain for computer science. Berndt and Teixeira [4] state that these applications generally target two different groups of users;

First, a broad target group primarily consists of the general public, educational professionals, ad- 
ministrators, and investors. The main focus for this group is on presentation. A second target group consists of the experts themselves (such as art historians, historians, archaeologists, restorers, and so on), who are concerned with the collection, preservation, exploration, and mediation of our cultural heritage.

It can be said that the first group does and will directly benefit from the advances in the following areas of computer graphics research; photorealistic and non-photorealistic rendering, real time rendering which focuses on rendering for interactive applications, and specialized graphics hardware such as graphics processing units. The second group, on the other hand, demand better and more reliable ways of conducting research using computer assisted tools. Advances in computer vision help to create more accurate and more robust 3D scanners, which in turn enable the creation of better 3D digital replicas of cultural heritage sites and artifacts. Similarly, advances in computational analysis and human-computer interaction allow the creation of more advanced application software that eases research.

\subsection{Overview on Heritage Applications}

One of the requirements for heritage applications that are made for analytical research is their need for high quality data. Godin et. al. [6], in their survey of scanning technologies for heritage applications, state that a high-resolution 3D model contains a wealth of information available for analysis, which in turn allows the study of fine details such as tool marks or surface texture.The use of $3 \mathrm{D}$ scanning in cultural heritage research has been successfully demonstrated in a number of projects to produce very detailed 3D models of sculptures, building, structures, and archaeological finds $[3,11,13]$. After a high detailed scan of an cultural heritage artifact has been obtained and verified the resulting 3D model can be used by architects, archaeologists, restorers, and art historians for their research and professional work. These software applications should be able to visualize and let the researcher work on such huge models interactively to be considered successful. ArcTron's ArchaeoCAD [1], a commercial application suite for cultural heritage researchers offers CAD tools for archeology. It allows archaeological measures on different scales to be carried out and create documentation for them. The measurement tools functionality are similar to $\mathrm{CH}$-Tools, however it offers a CAD like interaction framework to perform tasks and focuses more on reconstruction and documentation of scanned artifacts. Cavalieri [5], a computer-aided drafting system presented by Callieri et. al. in 2006, is a software application that semi-automatically produces large format prints of technical drawings called drafts of cultural heritage artifacts. Their system is designed to work on already available higly accurate 3D digital models and their target audience is restorers and archaeologists that have to rely on hand drawn technical drafts for part of their work. The proposed computer-aided approach is meant to augment and replace manual technical drawing, which the authors argue is 'time-expensive and heavily dependent on human contribution and skill'. The resulting system is able to produce accurate technical drawings of the artifact according to the researcher's wishes using standard 3D rendering techniques such as flat shading with per face normals or smooth shading with per vertex normals.

\section{Problem Statement}

Although the use of digitized models in cultural heritage research is beneficial, the adoption of such techniques is problematic because nearly all experts are trained for using traditional tools on physical artifacts. Both 3D scanning and 3D modeling requires familiarity with the hardware and software in order to produce digital models of required accuracy and detail for analysis and visualization tasks. As recent developments in 3D scanning technologies have made the digitization of artifacts affordable; the amount of digitized models available for research increases rapidly. Consequently the need for specialized software for cultural heritage research and practice on 3D models becomes more apparent. The problem with using existing 3D modeling software for cultural heritage research on digitized artifacts is that effectively using these programs needs additional expertise with the software and user interface which the researcher may lack. Such software are usually general purpose modeling tools or designed for digital content creation, architecture, or manufacturing and thus do not mimic the traditional way cultural heritage researchers work with artifacts. Therefore, practitioners of the field cannot easily transfer their expertise in the domain to new software tools without further education or specific guidelines [10]. Applications targeting specific tasks such as archaeological pottery reconstruction exist, but they are limited by their tight focus and cannot be easily extended to other domains in analytical cultural heritage research $[12,18]$. Similar problems arise in the medical field as well, where the disparency between computer tools and formal education methods is acknowledged. In the medical domain, the generally preferred solution to this problem is to present tools with a familiar interface based on their real world counterparts [17].

\section{Description of the System}

\subsection{Design Decisions}

$\mathrm{CH}$ Toolbox visualizes digitized models of artifacts in $3 \mathrm{D}$ and allows the user to analyze the pieces using a space- 
ball and mouse driven interface. Several designs were considered for the user interaction scheme of $\mathrm{CH}$ Toolbox. One important consideration was the scope of functionality, meaning both the number of distinct tools and the way these are presented to the user, that was suitable for $\mathrm{CH}$ Toolbox in order to make it as accessible as possible for cultural heritage researchers with differing backgrounds and computer skills. Grossman et. al. [7] consider the same issue in their digital tape drawing application, a computerized version of the technique commonly used by artists in car design, and say that although additional functionality similar to 3D modeling programs would be beneficial, the amount of functionality that can be introduced before tape artists reject it because of the perceived similarity with complicated $3 \mathrm{D}$ modeling software is an important question. The question is of similar importance in the design of $\mathrm{CH}$ Toolbox, and in the end we chose to present only a small subset of the possible functionality in order to keep our tools as simple and as close as possible to their real-life counterparts.

\subsection{Interaction Methodology}

The three main operations in $\mathrm{CH}$ Toolbox are camera control, model selection and manipulation, and tool utilization. Typically the user moves the camera to get an understanding about the object loaded into the environment and to find a suitable view for using the tool that is to be utilized next. Object selection and manipulation is useful in situations where more than one 3D model is visualized. Tool selection and utilization commonly comes only after the user is satisfied with the position and orientation of the camera and the 3D model. According to Balakrishnan and Kurtenbach [2], these actions can be categorized as either pragmatic or epistemic actions. All of camera control and some object manipulation are considered epistemic actions if they are done with the intention of increasing perception and cognition. All other actions, such as tool selection and utilization, done with the intent of getting closer to accomplishing certain goals of a task are considered pragmatic actions. Epistemic and pragmatic actions complement each other, thus they do not necessarily have to be in sequential order and can be conducted in parallel. Asymmetric bimanual interaction is a familiar human trait that lends itself well to such parallelized actions. Guiard [8] lay the theoretical foundations of skilled bi-manual action with his Kinematic Chain model in 1987. According to his model, humans accomplish many tasks with two hands complementing each other. He states three principals that govern the asymmetry of human bi-manual gestures:

\section{Right-to-Left Spatial Reference in Manual Motion,}

2. Left-Right Contrast in the Spatial-Temporal Scale of Motion,

\section{Left-Hand Precedence in Action.}

Guiard's terms refer to a right-handed person, thus the dominant hand is right hand and the non-dominant hand is the left hand. The following subsections explain these principals and their impact on our user interaction design.

Right-to-Left Spatial Reference Guiard's first principle is that in bi-manual motions of the right hand finds its frame of reference from the motions of the left hand. He gives the examples of handwriting and sewing for actions where the left hand orients and stabilizes the subject of the motion so that the right hand can perform its manipulating motion easier. This principle can also be observed with the spatial measurement tools that we adopted for use with digital models. A tape measure is used unrolling the tape with the right hand while the left hand stabilizes the starting end of the tape. A caliper is used by holding the object to be measured with the left hand while the right thumb carefully adjusts the jaws of the caliper to fit the object. The usage of a bordimeter is similar to handwriting, because the left hand stabilizes the paper in both cases.

Asymmetric Motion Scale The second principle states that the two hands operate in different scales during bimanual action both temporally and spatially. The left hand moves less frequently and makes comparatively larger movements than the right hand. We can again confirm this principle in the utilization of the spatial measurement tools selected for $\mathrm{CH}$ Toolbox. The right hand performs the fine adjustment of either the tool, by moving the measuring end of the tape, altering the jaw opening of the caliper, or the object, by positioning the rim sherd to fit the circle drawn on the bordimeter.

Left-Hand Precedence The last principle follows the first principle, and it states that in a human skilled bi-manual task, the left hand starts its action before the right hand. This principle is observed especially in tasks where the postural and manipulative roles of the hand are clearly separated, e.g. handwriting, sewing, driving a screw. Such a distinction between roles is also apparent in the usage of tapes and calipers, and consequently the principle of left-hand precedence can be observed in these actions. In the case of the tape measure, the left hand must place and hold the end of the tape before the right hand can unroll it. Similarly, in the case of caliper use, the left hand must position and hold the object to be measured before the right hand can position the caliper and adjust its dial to obtain a precise measurement. In the final case of bordimeter use, the left hand must hold the paper steady while the right hand moves the sherd to be fitted, and thus its actions must precede the actions of the right hand. 


\subsection{Input Device and Interaction Scheme}

Based on the initial analysis of the real world counterparts of the spatial measurement tools and their usage, we chose a bimanual interaction scheme for $\mathrm{CH}$ Toolbox, where the mouse is used to switch between tools, toggle visualization modes, select the artifacts to be manipulated, and precise utilization of the measurement tool and the spaceball is used to translate and rotate the selected artifacts or the $3 \mathrm{D}$ widget that represents the tool, and to control the camera (see Figure 1). As stated earlier, bi-manual interaction is a familiar human trait and increases productivity in 3D camera and object manipulation tasks by enhancing depth perception through motion $[9,2]$. Considering the additional benefits gained by parallelization of pragmatic and epistemic actions, such an interaction scheme is a good candidate for tasks that follow Guiard's theory of skilled bimanual interaction.

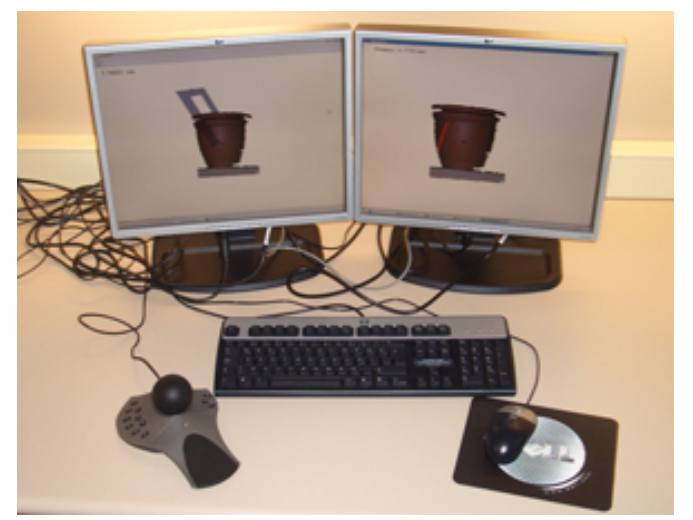

Figure 1. System setup showing the spaceball on the left (non-dominant hand) and the mouse on the right (dominant hand)

\section{Tools}

Spatial measurements and estimations based on these play an important part in the analysis of cultural heritage artifacts. In the next subsections, we consider the case of archaeological pottery reconstruction and three physical tools that are commonly used. These are the tape measure, caliper, rim chart, and area estimation. A discussion of the real tool along with its virtual counterpart is given. $\mathrm{CH}$ Toolbox is developed in $\mathrm{C}++$, using the open-source scenegraph library OpenSceneGraph [14] and runs in real-time on desktop PCs. We tested the system on an Intel Pentium D $2.8 \mathrm{GHz}$ PC with $1 \mathrm{~GB}$ RAM.

\subsection{Tape Measure}

The tape measure is used for determining the dimensions of a sherd and the surface distance between any two points on the sherd. The main purpose of the tape measure is to find geodesic distances either on the surface, along the rim, if the sherd is part of the rim, or along the fractured edges. The tool is used with two hands. The protrusion on the edge of the tape is fastened to one of the end-points of the distance to be measured, then it is held in place using one hand while the other hand pulls the measure and extends the tape to the other end-point. The measurement can be more easily read by locking the tape at the desired length. The tape measure is, in essence, an easier to use metered rope. The user fixes a point then moves the mouse to interactively visualize and measure geodesics originating from the start point as seen in Figure 2. The details of our algorithm and im-

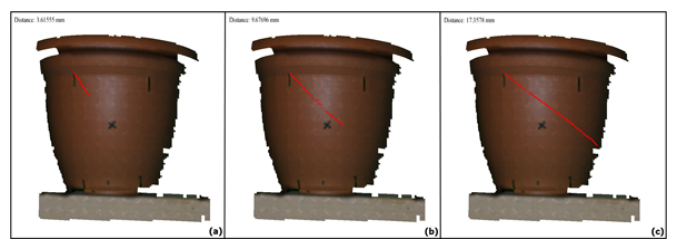

Figure 2. (a)The virtual tape measure is used by fixing the starting point and $(b, c)$ moving the end point with the mouse.

plementation can be found at [15]. For a sherd mesh with $17 \mathrm{~K}$ triangles, our tape measuring algorithm takes an average of 1.320 seconds for the preprocessing stage as seen in Table 1. The preprocessing was timed 100 times with each mesh. Although the performance is not adequate for realtime interaction, our interviews with cultural heritage experts show that the cost of the preprocessing is acceptable for real world use cases. The performance of the approxi-

Table 1. Mesh characteristics and preprocessing time for the tape measure tool

\begin{tabular}{l|rr|r}
\hline Model & Faces & Stn. points & Avg. time (s) \\
\hline Sherd & 17696 & 89859 & 1.320 \\
Sphere1 & 50986 & 255002 & 3.805 \\
Sphere2 & 204552 & 1023002 & 15.265 \\
David & 483498 & 2526815 & 1796.046 \\
\hline
\end{tabular}

mate geodesic algorithm is reported in Table 2. The results were obtained by timing the solution for 500 randomly selected start points with the sherd mesh and 100 points for 
the other three meshes. One approach to consider would be to let the user select the start and end points to calculate the geodesic distance between. This way, parts of the mesh not visible to the camera (either back-facing or out of the viewing frustrum) could be discarded before starting to solve the shortest path problem. Such a reducement in the number of vertices and edges would in turn reduce the computation time. However, this change would make the interaction scheme that mimics the real world usage of a tape measure not possible. In our case, even if the time between a user attaches the end of the virtual tape and the time the tape is operational again is long, the tool still behaves like a real tape measure after the shortest path problem is solved.

\section{Table 2. Performance of approximate geodesic algorithm}

\begin{tabular}{l|rrr}
\hline Model & Average time (s) & Median (s) & Std. dev. (s) \\
\hline Sherd & 1.84889 & 1.84788 & 0.23346 \\
Sphere1 & 4.36585 & 4.36408 & 0.11304 \\
Sphere2 & 18.14698 & 18.01450 & 0.69525 \\
David & 971.84767 & 1005.77050 & 140.31828 \\
\hline
\end{tabular}

\subsection{Radius Estimation}

A bordimeter, also called a rim chart, is a set of concentric circles drawn on a piece of paper or cardboard. It is used for estimating the rim radius of a vessel given a sherd belonging to the vessel's rim. Radius estimation is also used in volume, and consequently capacity estimation. Both assessments help in the classification of the vessel. The problem of fitting a circle to a given set of co-planar points is called $2 \mathrm{D}$ circle fitting. A best fit circle is computed and displayed interactively as the user moves the mouse over visualization of the artifact model as seen in Figure 3. The tool works as follows:

1. The plane defined by moving the mouse is intersected with the model to obtain a set of points on the plane.

2. The average of the points is taken as the initial estimate for the circle.

3. The circle is fit iteratively using least squares fitting to these selected points.

4. The center and the radius of the circle is computed.

Even if the least squares solution does not converge, the iteration for fitting the circle is stopped after a certain number of steps to maintain interactivity [15]. We found that

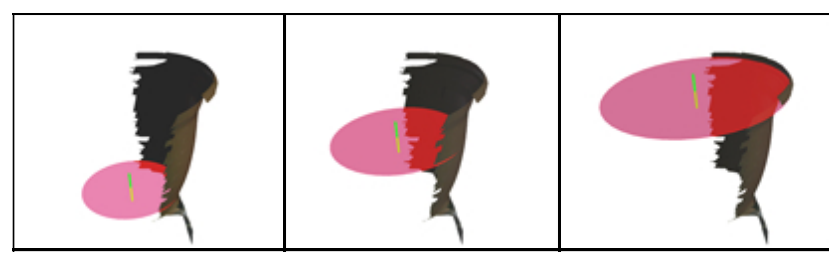

Figure 3. Screenshot of the virtual bordimeter in action

the solution converges in sufficient time if the points are not nearly linear. Otherwise, a warning message is displayed. This is not a problem with our test case since archaeological pottery has a curved surface. The circle and its center is visualized in addition to the text display of the location of the center and its radius because it helps the expert to visually verify the suitability of the numeric solution as the rotational axis of the artifact. During the measurement process the expert can use the spaceball to move and orient the model. She can also change the transparency of the circle visualization to prevent it from obscuring the artifact.

\subsection{Caliper}

The caliper is used for measuring the linear distance between an object's two opposite sides. The thickness of a sherd is useful in pottery analysis as it might give insights about the material and techniques used in making the pot as well as the intended usage of the vessel. Furthermore precise drawings and reconstructions of any artifact depend on precise measurements taken with a caliper. The tool is held with one hand, while the other hand manipulates the object to be measured. The tips of the caliper are than adjusted to get a firm touch on the surface of the object, after which a reading can be made. A too tight clamping action can deform both the caliper and the object depending on their material properties, which can yield an inaccurate measurement. We developed a virtual caliper based on the same idea of clamping the tips to the point of touching the measured object. The caliper is visualized as a semi-transparent plane that can be manipulated with the spaceball (see Figure 4). The plane has four cubes on the midpoints of each side, the top and bottom ones being fixed. The left and the right cubes are movable using the mouse wheel or the keyboard left and right arrows. These moveable cubes represent the tips of the caliper, which upon clicking get stuck in their relative positions. In a regular caliper only one tip is moveable, but in our virtual caliper implementation either the left, right or both tips can be adjusted. In our caliper implementation the closest face of the cube is checked for intersections with the model, with only one point of intersection meaning the tip has just touched the surface of the object. 


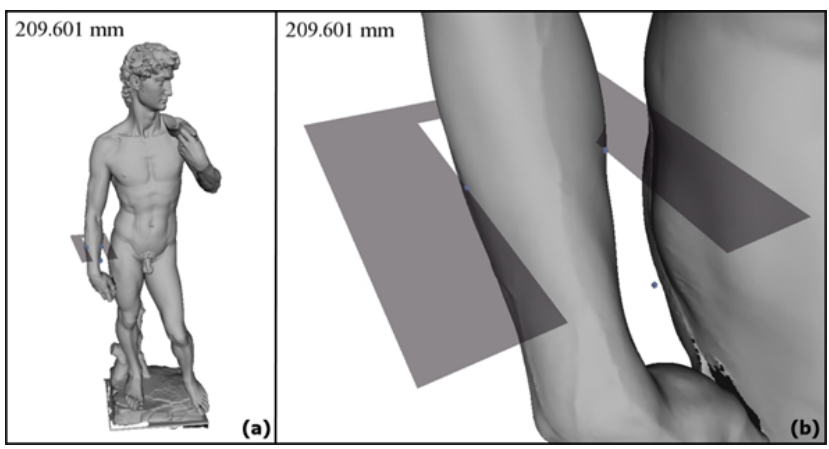

Figure 4. Screenshot of the caliper in action

\subsection{Area Estimation}

The purpose of the area tool is to estimate the surface area of a particular part of the digitized artifact specified by the user. Howerever, unlike the other tools presented the area estimation tool has no real-life counterpart. Therefore the interaction scheme for the tool was designed to be similar to other computer based selection schemes that would be familiar to the target user group. One interface element that almost all operating systems and software applications use to select multiple elements is called the selection rectangle. The usage of this elements employs rubberbanding, the effect that occurs when a shape is drawn on the screen and the shape resizes as the interface pointer is moved. As the name implies, the element acts like a rubber band that is stretched and hold in place with the use of a utensil. The same technique is also used commonly in graphics applications to draw a shape or select any part of an image or 3D model interactively. Consequently the rubberbanding technique was chosen for the area estimation tool based on the familiarity the user group would have with the scheme. After the selection rectangle is finalized by the user with the release of the mouse button, a set of rays originating from the eye point and passing through points on the rectangle present at a fixed interval is created. Each element of this set is then tested for a possible intersection with the triangles that make up the 3D model. The first intersected triangle for each ray, if it exists, is added to the surface to be summed to find the desired area. Finally the sum is calculated and displayed in the application. See Figure 5 for a close up of showing the intersected triangles and the intersection points. The computational complexity of this technique is $O(n m)$, where $n$ is the number of rays and $m$ is the number of faces to be tested for intersection. In first iteration the tool worked interactively, that is intersections were computed on each update of the selection rectangle, but this proved to perform unsatisfactory on huge meshes where the number of faces are near 500.000. The current version of the tool works by computing the intersection af-

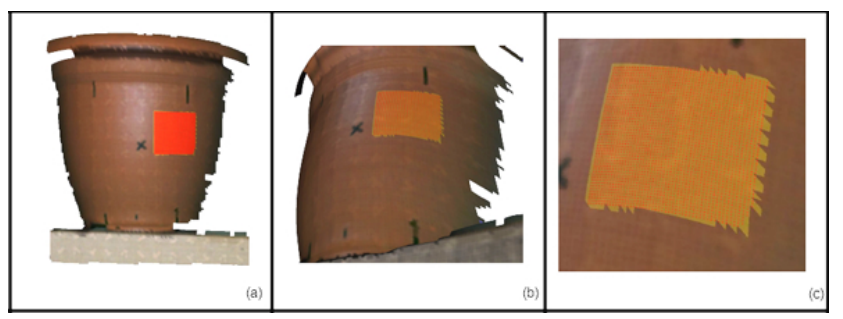

Figure 5. (a)A selection rectangle is used to fire of rays into the scene (b)The intersected triangles are colored yellow and the intersection points are colored red for clarification (c)Close-up of the selected area

ter the selection rectangle is finalized.

\subsection{Measurement Errors}

We compared rim estimations, surface distances, and caliper measurements taken on the real pot with the approximate results we got using our virtual tools on the digitized sherd mesh (Table 3). 5 points and 2 lines on the pot were marked before digitization in order to obtain enough reference points for measurement to find the error. Overall 17 spatial measurement were made 10 times each and then the differences between a real world measurement and its virtual counterpart were used to find the average relative error of the tools. The error rate of all the tools are near each other, $4.6 \%, 4.7 \%$, and $4.5 \%$. These similar percentages makes us suspect the underlying error of the scanning process dominates the error, thus making it impossible to reach a conclusion about the error of the approximation techniques without further tests using meshes digitized on systems with guaranteed precisions.

Table 3. Error rate of virtual tools

\begin{tabular}{l|rr}
\hline Tool & Avg. relative err. & Std. dev. \\
\hline Tape & $4.6 \%$ & $7.6 \%$ \\
\hline Caliper & $4.7 \%$ & $6.5 \%$ \\
\hline Bordimeter & $4.5 \%$ & $7.3 \%$ \\
\hline
\end{tabular}

\section{Discussion of Bi-manual Interaction Scheme}

The asymmetric bi-manual interaction scheme corresponds well with both the real world and the virtual versions of the tools implemented as part of the $\mathrm{CH}$ Toolbox software. The three principals stated by Guiard [8] that govern 
the asymmetry of human bi-manual gestures, right-to-left spatial reference in manual motion, left-right contrast in the spatial-temporal scale of motion, and left-hand precedence in action, is apparent in all the tools that make up the $\mathrm{CH}$ Toolbox. These principals are not exclusive and they can be observed together in the usage of a tool, albeit some of them could be more apparent within a certain task than others. Let us start by considering the example of utilizing a tape measure to find the spatial distance between two points on the surface of a pot. The sequence of actions in this scenario can be seen in Figure 6. The first action is the move-

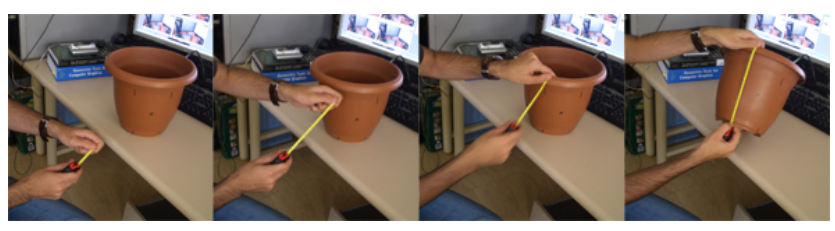

\section{Figure 6. A sequence of actions observed during tape measure utilization}

ment of the left hand to hold the end of the tape and then the movement of the hand to connect it with the point on the pot that the user wants to start the measurement from. This action confirms the third principle stating that the left hand precedes the right hand in starting the action. The next action is the movement of the right hand holding the tape itself to the desired point on the pot. The placement of the left hand on the pot and its fixation in space during this action comfirms the first principle, right-to-left spatial reference, as the left hand (end of the tape) clearly provides the right hand (the rolled tape itself) a reference in space that allows the unrolling of the tape. The final action in the sequence is the fine adjustment of the position of the right hand to accurately locate the desired end point on the pot. This action confirms the second principle that states a constrast in the spatial and temporal scales of motion between the hands. Comparing the distance covered by the left hand in one movement to locate the first point versus the distance covered by the right hand to locate the end point, we can observe the difference in the spatial scale of motion. The temporal difference in scale is also apparent, as the left hand has to stay motionless while the right hand makes several movements to unroll the tape and match the end point with a reading on the tape to obtain the desired measurement. The second tool implemented, a caliper, is used by holding the object to be measured with the left hand while the right thumb carefully adjusts the jaws of the caliper to fit the object. The first action is to pick up the object to be measured with the left hand while the right hand holds the caliper. This confirms the first third principle, left-hand precedence. The following action is the movement of the right hand to position the open jaw of the caliper to the de- sired area on the pot. Then the thumb on the right hand is utilized to move the wheel and thus adjust the opening between the ends of the tool (see Figure 7). This action is both slow and done in little increments in order to obtain a precise measurement. The said characteristics of motion of the right hand is a confirmation of the second principle, contrast in the spatial-temporal scale of motion between the hands. Finally, the left hand must hold the pot steady during measurement, which confirms the first principle that states the right-to-left spatial reference in manual motion. The last

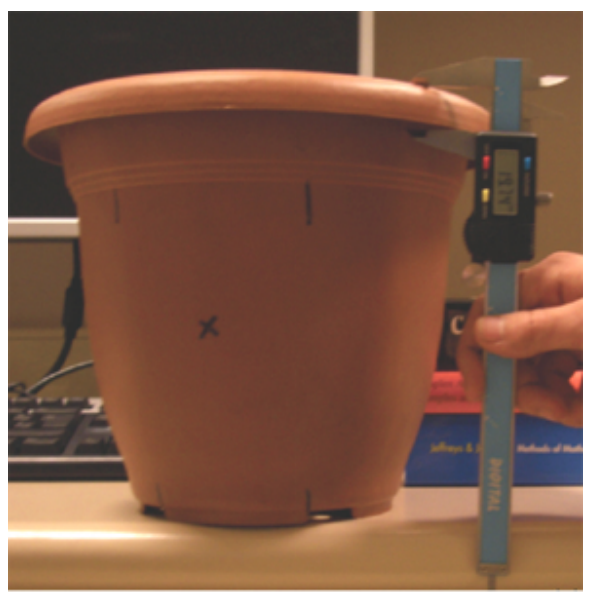

Figure 7. Caliper utilization in real life

tools, the rim-chart used to estimate the rim radius of a vessel and millimetric paper used to estimate an area on the surface of a pot, are both pieces of paper and thus the motions observed in their utilization show certain similarities. The starting action in their usage sequence is the positioning of the paper with the left hand. The rim-chart is placed on a flat surface and the millimetric paper is wrapped over the area to be measured. Despite this difference, the first action still confirms the third principle of left-hand precendence in both tools. The second action in rim-chart usage is the right-hand's placement of the pot or rim sherd on the paper and careful orientation of the piece in order to find a fitting circle segment. During the action of fitting the left hand can be used to orient the paper itself sometimes and to hold it steady other times (see Figure 8). This is done in order to help with the fitting task, and consequently confirms the first principle of right-to-left spatial reference. Furthermore, the differences in spatial and temporal scales between the hands in this task is a conformation of the second principle. Now the second action in the task of area estimation is the movement of the right hand holding a pen or pencil to mark the boundaries that correspond to the area to be measured under the millimetric paper. This action is similar to handwriting, but the paper must be securely held in place by the left hand in order to obtain a correct boundary. Here the 


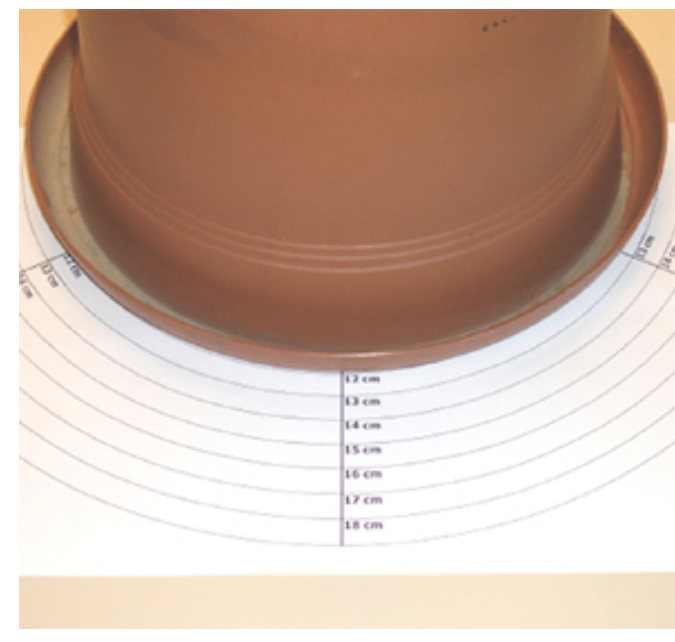

Figure 8. Rim-chart utilization in real life

left hand provides a spatial frame of refence to the marking hand, as predicted by the first principle. The underlying idea of the second principle is apparent if the actions of the right hand are observed. The pen must trace the boundary slowly and in little steps for correctness.

\section{Conclusions}

Our main contribution is the development of a platform for interactive computer-aided cultural heritage tasks. We implemented measurement tools that archaeologists daily use in real life by adapting algorithms developed for other computational geometry tasks. Another contribution is the introduction of bi-manual interaction to the cultural heritage domain, though possible benefits need to be further investigated with usability studies conducted with cultural heritage experts. The next step would be improving the accuracy of our tools, especially the radius estimation tool. We suspect the relatively high error rate is due to errors in the 3D scanning technology we used. Our tools should be tested with models obtained from other scanning technologies. We feel that the establishment of databases of peer-reviewed 3D models of cultural heritage artifacts will provide us with the opportunity to test our tools more throughly. $\mathrm{CH}$ Toolbox is released as an open-source application to aid researchers in the cultural heritage domain and can be found at [19].

\section{Acknowledgments}

This research is supported by Turkish National Science Foundation Research Grand 104E155. The authors would like to thank the Digital Michelangelo project for David model.

\section{References}

[1] ArcTron. Arccad. In http://www.arctron.com retrieved on May 2007.

[2] R. Balakrishnan and G. Kurtenbach. Exploring bimanual camera control and object manipulation in 3D graphics interfaces. In CHI '99: Proceedings of the SIGCHI conference on Human factors in computing systems, pages 56-62, New York, NY, USA, 1999. ACM Press.

[3] F. Bernardini, H. Rushmeier, I. M. Martin, J. Mittleman, and G. Taubin. Building a digital model of Michelangelo's Florentine Pietà. IEEE Computer Graphics and Applications, 22(1):59-67, 2002.

[4] E. Berndt and J. C. Teixeira. Cultural heritage in the mature era of computer graphics. IEEE Comput. Graph. Appl., 20(1):36-37, 2000.

[5] M. Callieri, P. Cignoni, R. Scopigno, G. Gori, and M. Risaliti. Beyond manual drafting: a restoration-oriented system. Journal of Cultural Heritage, 7:214-226, 2006.

[6] G. Godin, J.-A. Beraldin, J. Taylor, L. Cournoyer, M. Rioux, S. El-Hakim, R. Baribeau, F. Blais, P. Boulanger, J. Domey, and M. Picard. Active optical 3d imaging for heritage applications. IEEE Computer Graphics and Applications, 22:2436, 2002.

[7] T. Grossman, R. Balakrishnan, G. Kurtenbach, G. Fitzmaurice, A. Khan, and B. Buxton. Creating principal 3D curves with digital tape drawing. In $\mathrm{CHI}$ '02: Proceedings of the SIGCHI conference on Human factors in computing systems, pages 121-128, New York, NY, USA, 2002. ACM Press.

[8] Y. Guiard. Asymmetric division of labor in human skilled bimanual action: The kinematic chain as a model. Journal of Motor Behavior, 19(4):486-517, 1987.

[9] K. Hinckley, R. Pausch, D. Proffitt, and N. F. Kassell. Twohanded virtual manipulation. ACM Trans. Comput.-Hum. Interact., 5(3):260-302, 1998.

[10] H. E. II. Computer-assisted drafting and design: New technologies for old problems, 1988.

[11] M. Levoy, K. Pulli, B. Curless, S. Rusinkiewicz, D. Koller, L. Pereira, M. Ginzton, S. Anderson, J. Davis, J. Ginsberg, J. Shade, and D. Fulk. The digital Michelangelo project: 3D scanning of large statues. In SIGGRAPH '00: Proceedings of the 27th annual conference on Computer graphics and interactive techniques, pages 131-144, New York, NY, USA, 2000. ACM Press/Addison-Wesley Publishing Co.

[12] F. J. Melero, J. C. Torres, and A. Leon. On the interactive 3D reconstruction of Iberian vessels. In VAST 2003: 4th International Symposium on Virtual Reality, Archaeology and Intelligent Cultural Heritage, pages 71-78, Brighton, UK, 2003. EG Workshop Proceedings.

[13] D. Miyazaki, T. Oishi, T. Nishikawa, R. Sagawa, K. Nishino, T. Tomomatsu, Y. Takase, and K. Ikeuchi. The great Buddha project: modeling cultural heritage through observation. pages 181-193, 2001.

[14] OpenSceneGraph. http://openscenegraph.org.

[15] C. Ozmen and S. Balcisoy. A framework for working with digitized cultural heritage artifacts. In ISCIS 2006, volume 4263 of $L N C S$, pages 394-400. Springer Berlin / Heidelberg, 2006. 
[16] C. Ozmen and S. Balcisoy. A software system to work with 3D models in cultural heritage research. In A. DakouriHild and B. Frischer, editors, Beyond Illustration. Digital $2 D$ and 3D Technologies as Tools of Discovery in Archaeology, chapter 10. Archaeopress, in print.

[17] B. Preim, W. Spindler, K. J. Oldhafer, and H.-O. Peitgen. 3D distance measurements in medical visualizations. In Interactive Medical Image Visualization and Analysis, pages 31-36, 2001.

[18] M. S. Sagiroglu and A. Ercil. Automated assembly problem using an FFT based matching approach. In Signal Processing and Communications Applications, 2006 IEEE 14th, pages $1-4,2006$.

[19] C. Toolbox. http://graphics.sabanciuniv.edu/chtoolbox. 